\title{
The High Performance Shape Memory Effect (HP-SME) in Ni Rich NiTi Wires: In Situ X-Ray Diffraction on Thermal Cycling
}

\author{
Mauro Coduri ${ }^{1}$, Andrea Lausi ${ }^{2}$ and Ausonio Tuissi ${ }^{1, a}$ \\ ${ }^{1}$ CNR-IENI, National Research Council, Institute for Energetic and Interphases, Corso Promessi Sposi 29, Lecco, Italy
}

${ }^{2}$ Elettra - Sincrotrone Trieste S.C.p.A., S.S. 14 Km 163,5 in AREA Science Park IT-34149 Basovizza, Trieste (IT)

\begin{abstract}
A novel approach for using Shape Memory Alloys (SMA) was recently proposed and named highperformance shape memory effect (HP-SME). The HP-SME exploits the thermal cycling of stress-induced martensite for producing extremely high mechanical work with a very stable functional fatigue behaviour in Ni rich NiTi alloy. The latter was found to differ significantly from the functional fatigue behaviour observed for conventional SMA. This study was undertaken in order to elucidate the microstructural modifications at the basis of this particular feature. To this purpose, the functional fatigue was coupled to in situ Synchrotron Radiation X-Ray Diffraction, by recording patterns on wires thermally cycled by Joule effect under a constant applied stress ( $800 \mathrm{MPa}$ ). The accurate analysis the line profile XRD data suggests the accumulation of defects upon functional cycling, while the fibre texture was not observed to change. The functional fatigue exhibits a very similar behaviour as the line broadening of XRD peaks, thus suggesting the accumulation of dislocations as the origin of the mechanism of the permanent deformation
\end{abstract}

\section{Introduction}

SMA materials, and particularly the intermetallic NiTi compound, find a number of different industrial applications [1-4] thanks to their peculiar thermomechanical properties: the Shape Memory Effect (SME) and the Superelasticity (SE). The ability to recover high deformations is due to a Martensitic Transformation (MT), from a higher (austenite) to a lower (martensite) symmetry phase. The MT can be reversibly induced either by lowering the temperature or by applying a stress, exceeding a critical value, to the austenitic material.

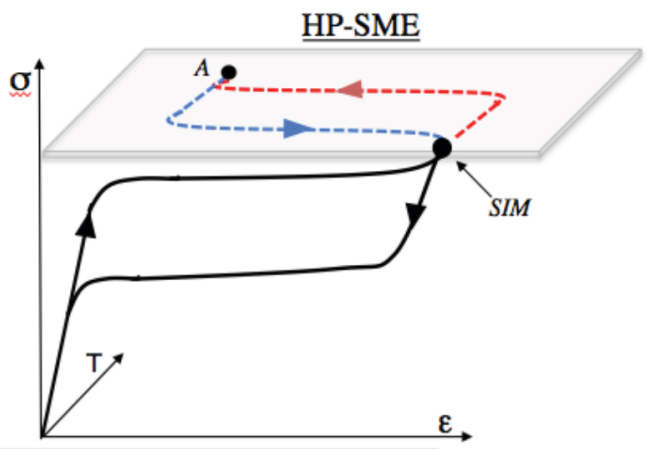

Figure 1. Sketch of the thermo-mechanical HP-SME path. Red and blue dashed lines stand for heating and cooling, respectively.
Recently, Ni-rich austenitic NiTi wires, were used to describe a new phenomenon named high performance shape memory effect (HP-SME) [5-6]. It consists in the thermal cycling of Stress Induced Martensite (SIM) and it allows achieving extremely high mechanical work (stresses of the order of $1 \mathrm{GPa}$ ), higher than that produced by conventional SMA actuators based on the heating/cooling of the de-twinned martensite [5-6]. A schematic view of HP-SME is depicted in Figure 1. In particular, when loading the austenite material above a critical stress, SIM is induced and the material deformation proceeds at almost constant stress. As the SIM is heated to restore austenite, under constant applied force, it shrinks back and the macroscopic deformation is therefore recovered up to point A in Fig.1. Finally, as the material is cooled down to RT, a complete SIM state is reproduced and the deformation is restored. Thus, throughout HP-SME thermo-mechanical path the large strain recovery is promoted at high stresses values; four times higher than the maximum applied stresses of SM actuator based on $\mathrm{Ti}$ rich, martensitic NiTi, which is limited to 250-300 MPa. In addition, HP-SME exhibits a functional fatigue behaviour well different with respect to conventional SME [5]: whereas the former is accompanied by a steep drop already within few cycles, conventional SME shows a smoother derive of the permanent deformation as highlighted in Figure 2.

\footnotetext{
${ }^{a}$ Corresponding author: tuissi@ieni.cnr.it
} 


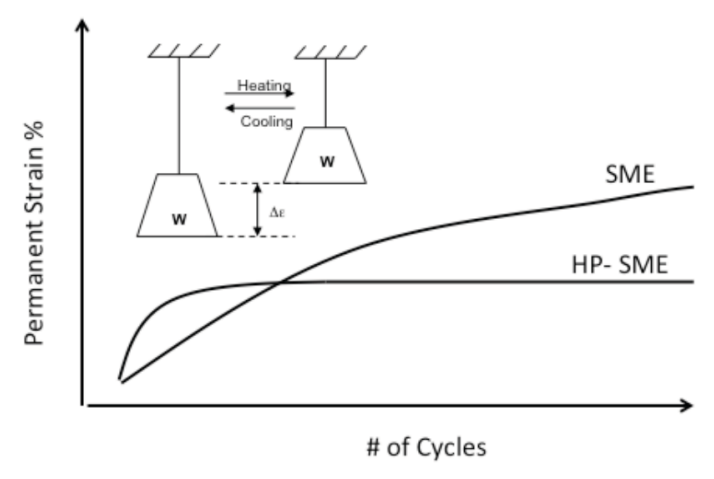

Figure 2. Schematic view of the permanent strain functional fatigue evolution of HP-SME compared to that of conventional SME materials [5].

The goal of the present study is then to point out the origin of the peculiar functional fatigue behaviour of materials subject to HP-SME. To this purpose, a structural characterization, to be performed in conditions as close as possible to those of the functional fatigue tests, is of the utmost importance. In this context, the requirement of mechanical loading to mimic typical application prevent conventional TEM characterization and make inconvenient X-ray structural measurements, at least by using conventional X-ray laboratory sources. To overcome this problem, we performed a combined fatigue and in-situ X-Ray Diffraction (XRD) study, the latter exploiting synchrotron radiation, aimed at assessing the typical microstructural evolution of NiTi wires under functional HP-SME cycling.

\section{Experimental}

XRD measurements were undertaken at the high resolution Materials Characterisation by X-ray diffraction (MCX) beamline of Elettra synchrotron (Trieste, Italy) in transmission mode with incident wavelength $\lambda=0.7500 \AA$, using a crystal analyser detector system.

The NiTi wire $100 \mu \mathrm{m}$ in diameter was horizontally positioned on the axis of the diffractometer thanks to an ad-hoc wire sample holder fixed to the diffractometer equipment, as illustrated in the experimental setup of Figure 3. The wire was tensioned by a constant load (weight) applied to the free end (right) of the SMA wire through a low friction pulley. The loads were selected in order to reach a complete SIM state at the room temperature. The loaded wires were then Joule heated applying a selected current values directed to the two current leads at the wire ends. In this context, data collected under current will be referred to as "high temperature" and those without applied current as "low temperature".

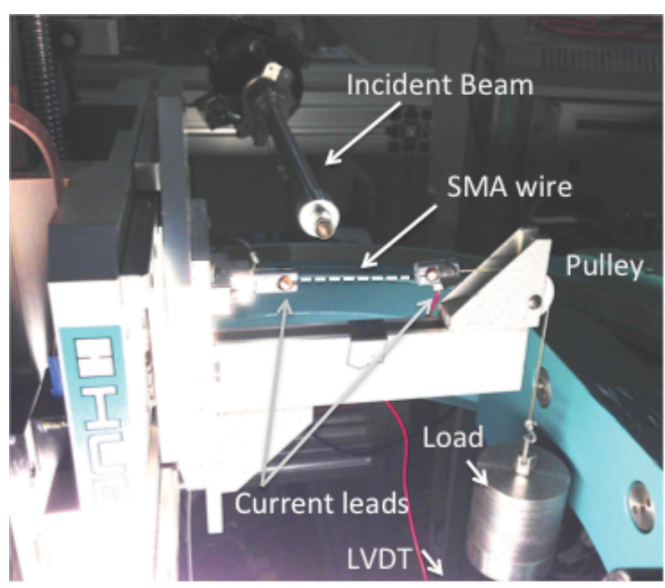

Figure 3. Image of the experimental apparatus employed to collect in-situ XRD patterns.

The displacement of the SMA wire as thermal cycling was measured by an Linear Variable Displacement Transducer (LVDT) detecting the position of the applied weight. A current source supplied 0.250 A for heating time of $10 \mathrm{~s}$, than it was switched off to restore low temperature SIM state; however during XRD data collection at high temperature the current was maintained for 1-2 $h$.

XRD patterns were collected after 1, 2, 5, 10, 20, 100 and 250 thermal cycles. Before each pattern collection, the sample had to be centred into the beam (beam size was roughly $300 \mu \mathrm{m}$ ), as during each transformation step the height of the wire changed.

The 13 to 402 theta range was selected in order to cover most of the expected austenite and martensite reflections. A large step point of about $0.05^{\circ}$ was selected to maximize signal-to-noise ratio, since the observed diffraction peaks were broad. The counting time, in the order of 1-2 hours per pattern, was varied for each of the samples according to their response.

\section{Results and Discussions}

\subsection{Fatigue tests}

The minimum and maximum wire lengths were measured by LVDT after cooling and Joule heating respectively and the permanent deformation $\Delta \mathrm{l} / \mathrm{l}_{0}(\%)$ after cooling was evaluated and plotted in Figure 4. As expected after \# 250 heating/cooling cycles under constant $800 \mathrm{MPa}$ applied stress, the wire subjected to HP-SME accumulated a permanent deformation over the first 2550 cycles, then a very flat functional fatigue curve exhibits less than $0.5 \%$ residual strain value for 50-250 cycles. For this heating current value $(0.250 \mathrm{~A})$ recoverable strains as large as $5 \%$ were evaluated on thermal cycling (curve not reported). 


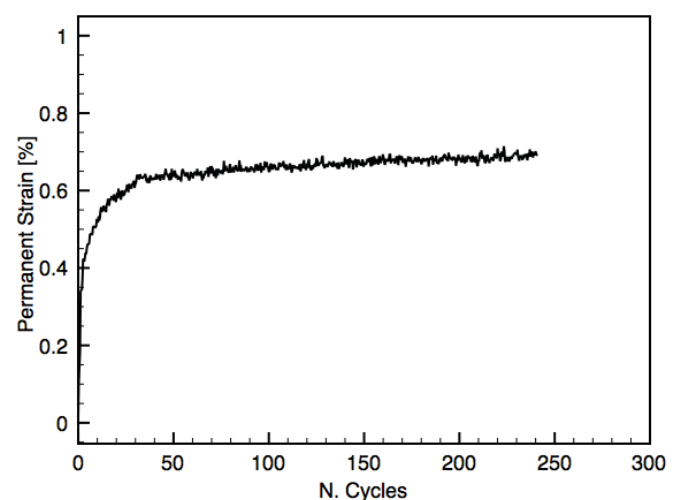

Figure 4. Permanent deformation for the 100 microns wire subjected to HP-SME with applied constant stress of $800 \mathrm{MPa}$ for \# 250 thermal cycles.

This behaviour confirms the finding of the work published elsewhere [5].

\subsection{XRD}

The experimental XRD patterns collected at high temperature as a function of the number of thermal cycles are shown in Figure 5. No evidence of phases different than austenite was probed. The main effect on the pattern induced by functional cycling concerns, instead, the peak broadening.

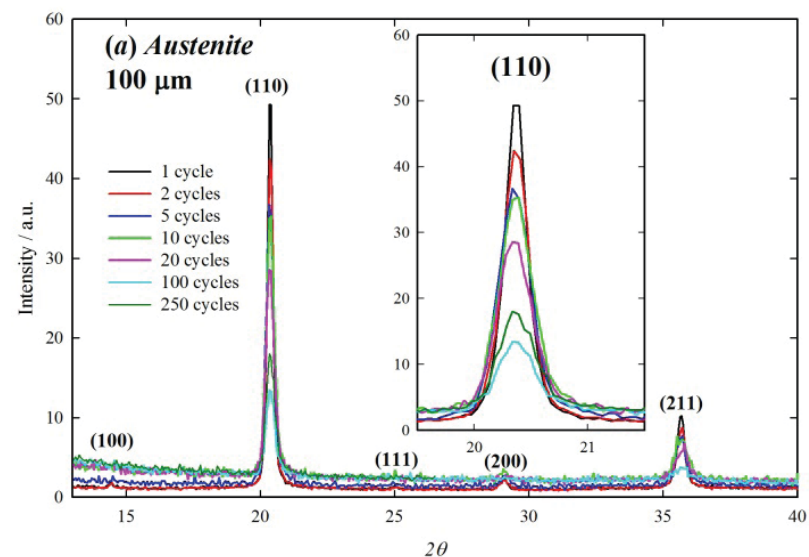

Figure 5. Evolution of XRD patterns at high temperature as a function of the number of thermal cycles. Miller indexes are shown close to the corresponding reflections.

Data were analysed after two different approaches: firstly the direct analysis, which corresponds to the fitting of the diffraction reflections of interest with a suitable PseudoVoigt function, has been undertaken to extract intensity, position and broadening as a function of the number of cycles. The latter parameter was described in terms of integral breadth, $\beta$. Then, the corrected data were subjected to Rietveld refinements for phase and texture quantification. The latter approach has the advantage to consider together all reflections of each phase, thus allowing the estimation of phase fractions and the quantification of the magnitude of the texture by means of a suitable texture index [7]. Based on the observed peak broadening and textures, the detection limit of martensite into austenite was estimated to be around $5 \%$. Instead, if we consider austenite as the secondary phase into martensite, its detection limit is around 3-5\%. As concerns the direct analysis, no detectable variation was observed in respect to the peak positions, therefore no apparent change in the lattice parameter was observed. The integral breadth, instead, shows a noteworthy variation. Its evolution with cycling with respect to the two main austenitic reflections, i.e. (110) and (211), are displayed in Fig. 2 in black and red full circles, respectively. The peak width increases during the first cycles and then it reaches a plateau within few tens of cycles. The broadening of diffraction peaks reflects a finite lattice coherence, generally related to size effects or the presence of other extended defects. In general, it is possible to match the peak broadening and the defect from which it originates, on the basis of the dependence of the broadening with the 2 theta angle.

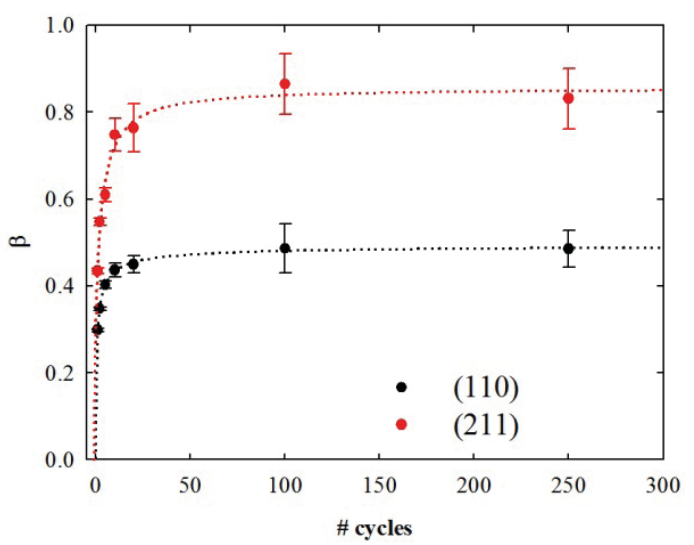

Figure 6. Integral breadth $\beta$ for reflections (110) (black) and (211) (red). Dotted lines (hyperbolic trend) are guides to the eye.

After the early works of Williamson and Smallman [8], the density of dislocations may be estimated on the basis of geometrical considerations. In the special case of a bcc lattice, considering the Burger vector along the $<111>$ direction and in the approximation of non-interacting dislocations, the dislocation density explicitly depends on the lattice parameter and on the increase of the integral breadth with the diffraction angle 2theta. Note that exactly the same approach was recently employed to calculate the dislocation density in NiTi wires under tensile stress [9].

The present data actually shows broadening with the diffraction angle, being the (211) reflection always much broader than (110), still this finding is only qualitative. Indeed, two reflections only have no statistical significance to quantify the dislocation density. Further investigations on the same samples (not reported here) confirmed the microstrain nature of the line profile 
broadening. Moreover, the characteristics of the system make more reasonable a mechanism of accumulation of dislocation, rather than a grain contraction, which in principle could return a similar effect on the pattern.

As concerns the texture, a first glance at the experimental patterns show no evident change in the relative intensity of the different reflections, which suggests no change in the texture upon cycling. This is confirmed by Rietveld analysis, which showed i) the increased intensity of (110) austenite and (020) martensite reflections with respect to a randomly distributed grain average; ii) a texture index, ranging within $1.55(10)$, invariant with the number of cycles. Moreover, being the monoclinic martensite obtained after the setting $a_{0} \cdot a_{0} \sqrt{2} \cdot a_{0} \sqrt{2}$, where $a_{0}$ stands for the lattice parameter of cubic austenite; the (110) and (020) reflection of austenite and martensite, respectively, correspond to the same family of planes. This is sketched in Figure 7. As a consequence, no evident change of texture, arising neither from austenite to martensite transformation, nor from the functional cycling, were found to occur during HP-SME.
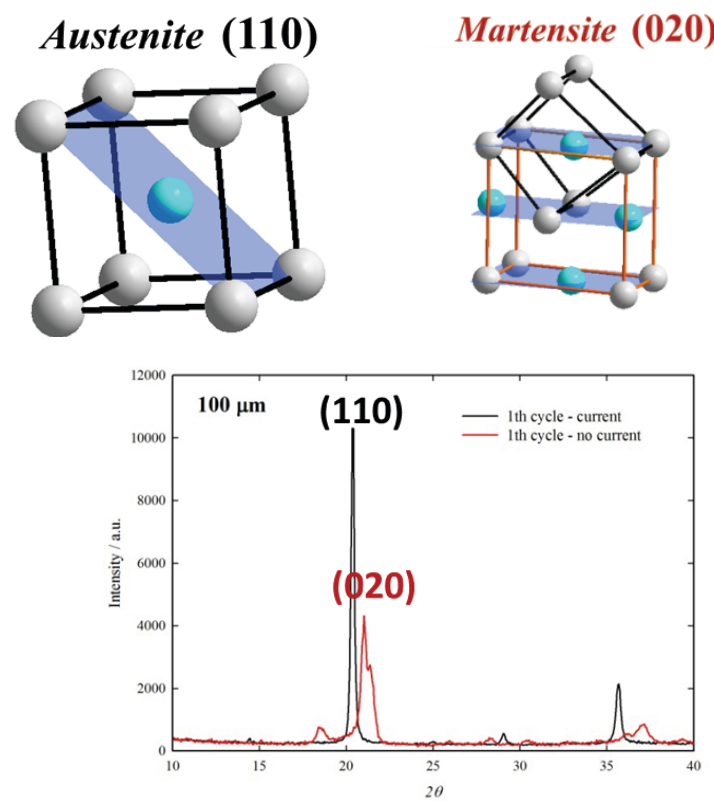

Figure 7. Sketch of (110) reflection of austenite (top, left), (020) reflection of martensite (top, right). The austenite-like cuboid is displayed within the martensite structure in order to account or the change of setting. The bottom panel shows austenite (black) and martensite (red) patterns a recorded during the first thermal cycle.

\section{Conclusions}

The functional cycling of NiTi wires strongly affects the defectivity of the material. This is made evident by the systematic reflection broadening of XRD peak profiles with increasing the number of cycles. This behaviour suggests the accumulation of dislocations in the material, which saturates within some tents of thermal cycles. A very similar dependence with the number of cycles is observed in the case of the permanent deformation, recorded during the same XRD in situ investigation.

This finding suggests a close interplay between the increase in the dislocation density, qualitatively probed by in-situ XRD, and the steep increase of permanent deformation, both of them occurring within a few tens of cycles.

\section{References}

1. K. Otsuka, C.M. Wayman, Shape Memory Material, (Cambridge University Press, 1998)

2. J. Van Humbeeck, Mater. Sci. Eng., A 273 (275) (1999) 134-148.

3. T. Duerig and A. Pelton, D. Stockel, Mater. Sci. Eng., A 273 (275) (1999) 149-165.

4. A. Biscarini, G. Mazzolai, A. Tuissi, Enhanced Nitinol Properties for Biomedical Applications, Recent Patents on Biomedical Engineering, 1, 180, (2008)

5. R. Casati, M. Vedani, A. Tuissi, Scripta Mater. 80, 13 (2014)

6. R. Casati, C.A. Biffi, M. Vedani, A. Tuissi, Functional Materials Letters, Vol. 7, No. 5 (2014) 1450063

7. R.B. Von Dreele, J. Appl. Cryst. 30, 517 (1997)

8. G.K. Williamson, R.E Smallman, Phil. Mag. 1, 34 (1956)

9. E. Polatidis, N. Zotov, E. Bischoff, E.J. Mittemeijer, Scripta Mater. 100, 59 (2015) 